\title{
Correction to: Human migration: the big data perspective
}

\author{
Alina Sîrbu ${ }^{1} \cdot$ Gennady Andrienko ${ }^{2,3} \cdot$ Natalia Andrienko ${ }^{2,3} \cdot$ Chiara Boldrini $^{4} \cdot$ Marco Conti $^{4} \cdot$ Fosca Giannotti $^{5}$. \\ Riccardo Guidotti ${ }^{5} \cdot$ Simone Bertoli $^{6} \cdot$ Jisu Kim $^{7}$. Cristina loana Muntean ${ }^{5} \cdot$ Luca Pappalardo $^{5} \cdot$ Andrea Passarella $^{4}$. \\ Dino Pedreschi ${ }^{1} \cdot$ Laura Pollacci $^{1} \cdot$ Francesca Pratesi $^{1} \cdot$ Rajesh Sharma $^{8}$
}

Published online: 21 May 2021

(c) Springer Nature Switzerland AG 2021

\section{Correction to: \\ International Journal of Data Science and Analytics (2021) 11:341-360 \\ https://doi.org/10.1007/s41060-020-00213-5}

The article "Human migration: the big data perspective", written by Alina Sîrbu, Gennady Andrienko, Natalia Andrienko, Chiara Boldrini, Marco Conti, Fosca Giannotti, Riccardo Guidotti, Simone Bertoli, Jisu Kim, Cristina Ioana Muntean, Luca Pappalardo, Andrea Passarella, Dino Pedreschi, Laura Pollacci, Francesca Pratesi, Rajesh Sharma originally published electronically on the publisher's internet portal (currently SpringerLink) on April 10, 2021 without

The original article can be found online at https://doi.org/10.1007/ s41060-020-00213-5.

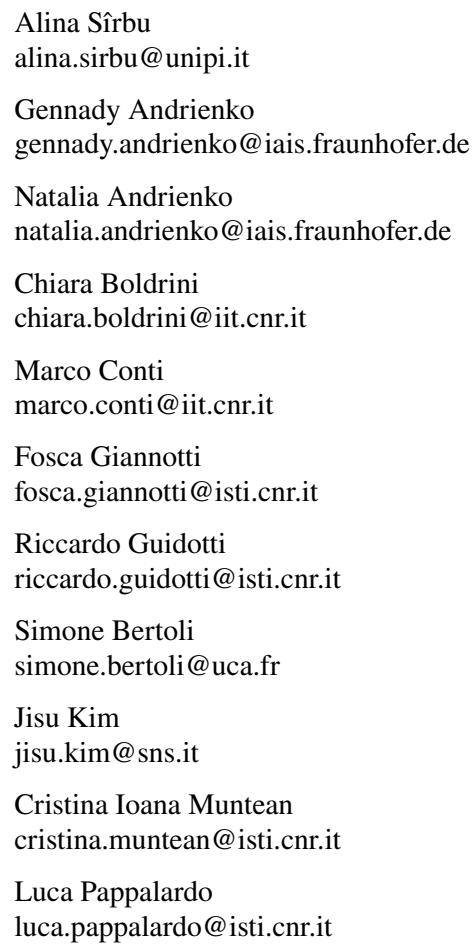

